\title{
INEQUALITIES RELATED TO THE GEOMETRIC MEAN OF ACCRETIVE MATRICES
}

\author{
Juntong LiU, Jin-Jin Mei And DengPeng ZHANG*
}

Abstract. We present some inequalities related to the recently defined geometric mean of two accretive matrices. Firstly, we show that if the block matrix $\left(\begin{array}{cc}A & X \\ Y^{*} & B\end{array}\right)$ is accretive, then the singular values of $(X+Y) / 2$ are weakly log majorized by the singular values of the geometric mean of $A$ and $B$. This extends a result of M. Lin.

Mathematics subject classification (2020): 15A42.

Keywords and phrases: Geometric mean, accretive matrix.

\section{REFERENCES}

[1] T. Ando, Geometric mean and norm Schwarz inequality, Ann. Funct. Anal. 7 (2016) 1-8.

[2] T. Ando, Hua-Marcus inequalities, Linear Multilinear Algebra 8 (1980) 347-352.

[3] R. Bhatia, Matrix Analysis, GTM 169, Springer-Verlag, New York, 1997.

[4] R. Bhatia, Positive Definite Matrices, Princeton University Press, Princeton, 2007.

[5] S. DRURY, Principal powers of matrices with positive definite real part, Linear Multilinear Algebra 63 (2) (2015) 296-301.

[6] L. KUAI, An extension of the Fiedler-Markham determinant inequality, Linear Multilinear Algebra 66 (2018) 547-553.

[7] E.-Y. LEE, The off-diagonal block of a PPT matrix, Linear Algebra Appl. 486 (2015) 449-453.

[8] M. Lin, The Hua matrix and inequalities related to contractive matrices, Linear Algebra Appl. 511 (2016) 22-30.

[9] M. LIN, F. SUN, A property of the geometric mean of accretive operator, Linear Multilinear Algebra 65 (2017) 433-437.

[10] M. Lin, Some inequalities for sector matrices, Oper. Matrices 10 (2016) 915-921.

[11] M. LIN, Inequalities related to $2 \times 2$ block PPT matrices, Oper. Matrices 9 (2015) 917-924.

[12] M. Raissouli, M. S. Moslehian, S. Furuichi, Relative entropy and Tsallis entropy of two accretive operators, C. R. Acad. Sci. Paris, Ser. I 355 (2017) 687-693.

[13] X. Zhan, Matrix Theory, GSM 147, American Mathematical Society, Providence, RI, 2013.

[14] F. Zhang, Matrix Theory: Basic Results and Techniques, second edition, Springer, New York, 2011. 